\title{
AiMT
}

Advances in Military Technology

Vol. 14, No. 2 (2019), pp. 197-212

ISSN 1802-2308, eISSN 2533-4123

DOI 10.3849/aimt.01293

\section{Hydration Bags Construction Materials Resistance to Chemical Warfare Agents and their Comparison}

\author{
J. Kiszka* and S. Florus \\ NBC Defence Institute, University of Defence, Vyškov, Czech Republic \\ The manuscript was received on 7 February 2019 and was accepted \\ after revision for publication on 21 June 2019.
}

\begin{abstract}
:
When working in a contaminated area while wearing individual protective equipment, there is a high risk of overheating and dehydration. In accordance with the valid documents of the Army of the Czech Republic, a person using protective equipment has to be provided with an adequate fluid intake. To perform the work, it is necessary to equip the soldier with a hydration device that is resistant to chemical warfare agents and is structured in a way that allows its use when means of personal protection or other equipment is used. The aim of the experimental work was to verify resistance of the construction materials of hydration devices and to compare them. For this purpose, devices designed to provide resistance to chemical warfare agents and those that did not provide it were tested. To determine breakthrough times of selected materials, the MIKROTEST and the MINITEST methods were used.
\end{abstract}

\section{Keywords:}

breakthrough time, chemical warfare agents, hydration device, mustard gas, organism dehydration

\section{Introduction}

It is highly stressful for a person to work when individual protective equipment is used [1]. First of all, there is the issue of mental stress which stems from the fact that the work itself is performed in a hazardous environment from which the person is separated only by protective equipment that is used. In this case, confidence in the quality of the protective equipment, meaning its resistance to the current contaminant, and in its proper functioning and intactness is an essential prerequisite for the person's willingness to even take the risk of working in a contaminated area. The willingness to work in a contaminated area is also dependent on the person's knowledge of both the properties of the contaminant and its potential effects on organism and the protective

\footnotetext{
* Corresponding author: NBC Defence Institute, University of Defence, 68201 Vyškov, Czech Republic.E-mail: jkiszka@seznam.cz.
} 
properties of the individual protective equipment itself. Another factor that has a profound effect on a person is heat stress. It is caused by partial or total insulation of body by means of individual protection and the impossibility of their modification, by weight of the individual protective equipment itself, creating an additional load for its users, and by energy expenditure attributable to the work performed that is made more difficult by the necessity to use individual protective equipment, as well as by the issue of mental stress. The physical condition of the user and the ambient temperature also play their role in the process. Stress associated with the use of means of individual protection leads to the loss of organism fluids as a result of sweating, a mechanism designed for the purpose of organism cooling and thereby preventing its overheating. Lack of fluids in the organism, which can occur within several dozens of minutes when means of individual protection are used, is called acute dehydration for which the loss of one or more per cent of body weight is typical [2-4]. Despite hydration awareness and existence of recommended amounts that have to be provided for organism under given temperature conditions and when using certain means of protection or their combination [5], its implementation in a contaminated area can be faced with difficulties which are associated with technical and safety aspects of the process.

At present, two fundamental means of organism hydration can be used in a contaminated area, provided that the face mask is equipped with a fluid intake device a field bottle with a special stopper and a hydration bag with a connector enabling the connection between the bag and the fluid intake device of the face mask. The field bottle has certain disadvantages - small volume, usually of about 0.81 , quite lengthy, relatively difficult and not always safe handling with the field bottle stopper when attaching the fluid intake device, and discontinuous fluid intake. Hydration bags, on the other hand, can have volume of up to several litres, which is sufficient for work lasting for several hours; they can be permanently attached to the intake valve of the face mask, and they can enable continuous fluid intake. They have a certain disadvantage and that is their size and constructional arrangement which could be a limiting factor in the use of the hydration bag in connection with other essential equipment necessary for work in contaminated areas.

However convenient the use of hydration bags may be, the condition of their resistance to anticipated harmful substances to which they will be exposed has to be fulfilled. Hydration bags do not necessarily have to be resistant if they are worn under clothing and if protection against contaminants in the external environment is ensured by means of protective clothing. To achieve this, it is also possible to use resistant cases that would protect the fluid container so that they could be worn on the means of protection.

Currently, there is a wide range of hydration bags available on the market. The simplest hydration bags consist of a fluid container with an opening with a screw cap and a seal and a suction tube with a tap and an intake valve. In order to use the hydration bag, it has to be inserted into a regular backpack. For military needs, hydration devices make up a set which consists of a backpack and a hydration bag with a sealable opening, a suction tube with a tap and an intake valve. Constructional arrangement and construction material resistance then depends on whether the hydration device is designed for work in environment contaminated by chemical warfare agents or not.

As for the construction material resistance of the hydration bags available on the market, major manufacturers offer bags with high CBRN resistance (chemical, biological, radiological and nuclear). However, there are no resistant cases for hydration 
bags without protection against CBRN agents. If such cases were to be used, it would be necessary to develop them specifically for such a purpose.

The work focuses on evaluating the properties regarding resistance of selected hydration bags against chemical warfare agents by determining the breakthrough time against sulphur mustard. The aim is to determine whether the materials used to construct hydration bags possess the resistance against chemical warfare agents and if so, to determine the exact values of such resistance. For this purpose, three available hydration bags with a volume of 31 were chosen:

- the hydration bag used in the Army of the Czech Republic (the CamelBak hydration bag made from polyurethane carried in the backpack manufactured by S.P.M. Liberec), CBRN non-resistant [6],

- the Source Gear Tactical 3L hydration bag made from polyethylen, used on regular basis for 4.5 years [7],

- the Source MAX CBRN 3L hydration bag made from mixture of polyethylene and polyurethane, CBRN resistant [8].

All hydration bags have similar basic construction - they consist of a hydration bag with a suction tube inserted in a backpack (Fig. 1 shows the Source MAX CBRN 3L hydration bag as an example).

The reason why the Source Gear Tactical 3L hydration bag was used was to determine whether it would be possible to use the worn-out bag in a contaminated area even though the material was visibly damaged and to determine its residual protective properties. Unfortunately, the same unused bag was not available, thus it was impossible to determine the exact impact of the wear. Yet, such results are still relevant.

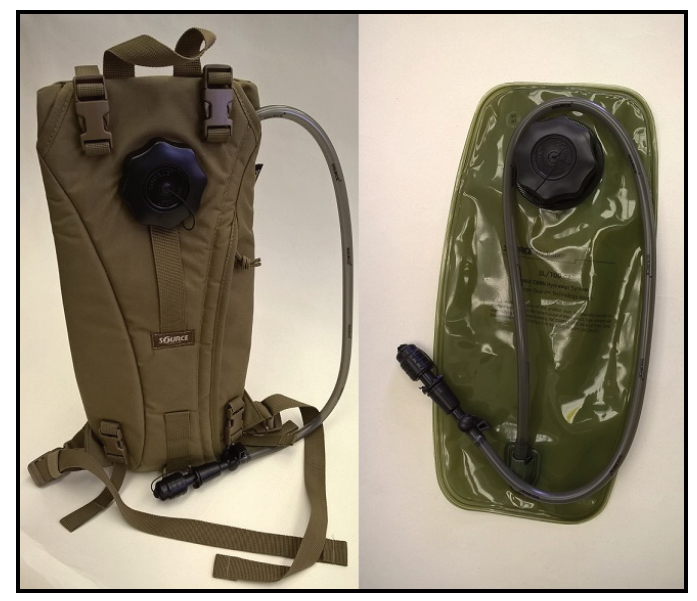

Fig. 1 The Source MAX CBRN 3 L hydration bag

\section{Experimental Part}

\subsection{Chemicals Used}

To conduct the experimental part, sulphur mustard [bis(2-chloroethyl)sulphide, $89.9 \%$, VOZ 072 Zemianské Kostolany, Slovakia] was used to determine resistance of the examined material against chemical warfare agents. The $1.5 \%$ solution of the CNITI- 8 
chloramine [ $N$-chloro- $N$-(2-tolyl)benzamide, self-produced] in carbon tetrachloride (99\%, Merck) was used in combination with Congo red (p.a., Merck) to detect sulphur mustard that permeated to the other side of examined material. For decontamination, the $10 \%$ water-ethanol $(1: 1)$ solution of chloramine $\mathrm{T}$ [sodium $\mathrm{N}$-chloro-4toluenesulphonamide, $81 \%$, Bochemie) was used.

\subsection{Equipment and Devices Used}

To conduct the experiment, the following equipment was used:

- the FRIOCEL 111 biological incubator (BMT Medical Technology s.r.o., Brno, Czech Republic),

- the DESAGA SG1B spray (Desaga GmbH, Wiesloch, Germany),

- the Mitutuyo ABSOLUTE micrometer (Mitutuyo Corporation, Kawasaki, Japan),

- products for the MIKROTEST method containing two pieces of cover slips with the dimensions of $(70 \times 70) \mathrm{mm}$ and a thickness of $2 \mathrm{~mm}$, a rubber mask made from bromobutyl rubber with the dimensions of $(70 \times 70) \mathrm{mm}$ and a thickness of $2 \mathrm{~mm}$ with a central hole of $20 \mathrm{~mm}$ in diameter and two MAULY $25 \mathrm{~mm}$ clamps,

- products for the MINITEST method containing a cover slip with the dimensions of $(180 \times 130) \mathrm{mm}$ and a thickness of $2 \mathrm{~mm}$, a metal mask with the dimensions of $(180 \times 130) \mathrm{mm}$ and a thickness of $2 \mathrm{~mm}$ with twenty holes of $20 \mathrm{~mm}$ in diameter, a PVDF desk (polyvinylidenf) with the dimensions of $(180 \times 130) \mathrm{mm}$ and a thickness of $10 \mathrm{~mm}$ with twenty holes of $20 \mathrm{~mm}$ in diameter drilled into mid-thickness of the desk into which glass frits are inserted and four MAULY $25 \mathrm{~mm}$ clamps, white paper towels that produce neutral leachates.

\subsection{Measurement Methods Used}

In order to measure resistance of the construction materials, two methods were used. Whereas the MIKROTEST method [9] was used to measure their resistance to liquid mustard gas, the MINITEST method [10-13] was used to measure their resistance to mustard gas in form of vapours. For the detection of mustard gas, both methods use an indicator paper which is made from sufficiently thick white paper that produces neutral leachates and is impregnated with a water-ethanol solution (1:1) of Congo red with the concentration of $0.1 \%$ and with a $1.5 \%$ solution of CNITI- 8 [ $N$-chloro- $N$ - $(2-$ tolyl)benzamide] in carbon tetrachloride. Thus, the prepared detection paper is redcoloured.

The detection reaction takes place in two steps. Firstly, mustard gas reacts with CNITI-8 to form hydrogen chloride which then reacts with the Congo red acid-base indicator to produce a blue colour in the place of the mustard gas permeation. For the purpose of measuring resistance of the hydration bag suction tubes, a new methodology using the same mustard gas detection mechanism as the MIKROTEST and the MINITEST methods was developed. The measuring setup itself is currently the subject of assessment, and there is an assumption that the given methodology will be part of recognized methodology for the suction tube resistance measurement. For this reason, any more details concerning the measuring setup are not provided at this point. 


\subsection{Preparation of Samples}

In order to obtain compact results of chemical resistance of the hydration bags construction materials for the MIKROTEST and the MINITEST methods, samples were cut out in such a way so that they would be intact and homogeneous, i.e. they would not include reinforcement of the polymeric parts and would be of the same visual quality. In total, six sample types were prepared:

- a film of the hydration bag for the measurement of effects of liquid and gaseous chemical warfare agents,

- an outer fabric layer of the backpack,

- a combination of an outer fabric layer of the backpack - an insulating layer an inner fabric layer of the backpack,

- a combination of an outer fabric layer of the backpack - an inner insulating layer - an inner fabric layer of the backpack - a film of the hydration bag,

- as for the hydration bag of the Army of the Czech Republic - an intersewn combination of an outer fabric layer of the backpack - an inner insulating layer - an inner fabric layer of the backpack,

- a suction tube of the hydration bag.

For the MIKROTEST method, the maximum number of samples from each hydration bag with dimensions that enable to measure chemical resistance in a reliable manner was prepared. For the MINITEST method, which is used to measure resistance to gaseous chemical warfare agents, samples were prepared to enable to perform the measurement on the largest number of measurement spots out of twenty on the PVDF desk into which a testing chemical, i.e. mustard gas, was dosed. The tubes were cut in the lengths of $60 \mathrm{~mm}$, and for the purpose of measurement, they were used without their outer fabric layers which are not relevant for the measurement of their chemical resistance as they protect the tube against mechanical damage such as abrasion. All the samples were numbered, and their thickness was measured. Since it was not possible to uncurl the tubes and use standard measuring methods, their external diameter was measured instead of wall thickness.

\subsection{Processing of Results}

The thickness measurement results and the breakthrough time results were statistically processed using the MS Excel software. To test the robustness of the measurement results, a normality test of all datasets was performed (the Shapiro-Wilk normality test). If the results were normally distributed, the dataset was further tested using the Grubbs 'test for outliers. Otherwise, the Dean-Dixon test for outliers, which is used when the distribution is different than normal or unknown, was performed. Since the minimum values of both the thickness and the breakthrough times from the particular measurement sets are significant for individual protection, minimum values of these measurements were compared as the difference between the mean value and the value of the $95 \%$ confidence interval.

Thickness of the CamelBak hydration bag film was only apparent because the film surface was not smooth but relief-like (Fig. 2). Thickness of the fabric layers and inner insulating layers should be also considered as indicative. 


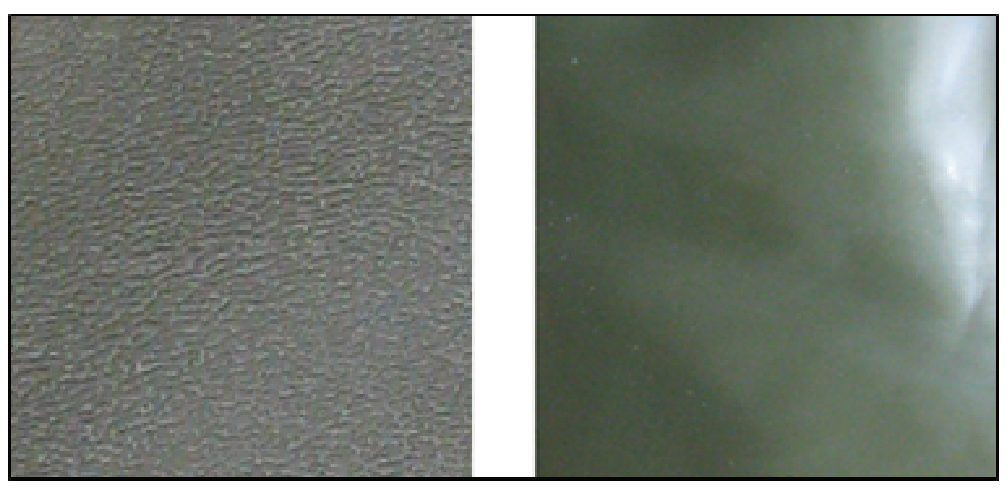

a)

b)

Fig. 2 Comparison of hydration bags film surface

a) CamelBak, b) Source Tactical $3 L$

\section{Results and Discussion}

The resistance of the Source Tactical 3L and Source MAX CBRN 3L hydration bags to chemical warfare agents was unknown. For this reason, their resistance to mustard gas was measured at a temperature of $60{ }^{\circ} \mathrm{C}$ which was recalculated to a temperature of $30{ }^{\circ} \mathrm{C}$. Dependence, according to which a temperature increase of $10{ }^{\circ} \mathrm{C}$ decreases the resistance of polymeric construction materials to a half of that at a temperature of $10{ }^{\circ} \mathrm{C}$ lower, was employed. This means that at a temperature of $60{ }^{\circ} \mathrm{C}$, the breakthrough time should be eight times shorter than at a temperature of $30^{\circ} \mathrm{C}$. Although such resistance is only estimated, practical experience has shown that this estimate can be taken as the basis for further experimental work. The measurement results have shown that at a temperature of $60{ }^{\circ} \mathrm{C}$, the breakthrough time of the Source Tactical 3L hydration bag was $28 \mathrm{~min}$, which would amount to $224 \mathrm{~min}$ at a temperature of $30^{\circ} \mathrm{C}$. Contrastively, the breakthrough time of the Source MAX CBRN 3L hydration bag was $287 \mathrm{~min}$ at a temperature of $60{ }^{\circ} \mathrm{C}$, which would amount to $2296 \mathrm{~min}$ at a temperature of $30{ }^{\circ} \mathrm{C}$. Following the period of $287 \mathrm{~min}$, the measurement was stopped considering that the breakthrough time would be more than $38 \mathrm{~h}$, which is an unrealistic period of time for a stay in a contaminated area and a continuous use of the bag as well. At a temperature of $60{ }^{\circ} \mathrm{C}$, it was apparent that the film of this hydration bag was swelling.

The Source MAX CBRN 3L hydration bag had the largest minimum polymeric film thickness, $0.425 \mathrm{~mm}$ specifically, followed by the Source Tactical 3L hydration bag with a thickness of $0.415 \mathrm{~mm}$ and the CamelBak hydration bag with a thickness of $0.407 \mathrm{~mm}(0.409 \mathrm{~mm}$ without outliers). It is possible that the uneven surface of the CamelBak hydration bag (Fig. 2a) and fluctuations in thickness values had practical implications on its chemical resistance. Measurement of breakthrough times for liquid mustard gas using the MIKROTEST method has shown that CamelBak is the least resistant to mustard gas, which could have been anticipated since this bag is not designed for work in an area contaminated by chemical warfare agents. Its breakthrough time was 47.4 min (Fig. 3). The breakthrough time of the Source Tactical 3L hydration bag was 169.9 min, meaning less than was expected from the indicative measurement. 
It has to be noted that the bag had been in use for 4.5 years which could have had an impact on the resulting breakthrough time.

As seen in Fig. 3, the breakthrough time of the Source MAX CBRN 3L was $3060 \mathrm{~min}$ which equals $51 \mathrm{~h}$; however, this value is not final due to the fact that the measurement was stopped after this period of time with no permeation being observed. The results of the indicative measurement were thereby confirmed. Following the measurement of the hydration bag breakthrough times, functionality of the indicator paper was checked by carefully disassembling the device used for measurement and removing the indicator paper and exposing it to the effects of mustard gas vapours. By turning blue, the functionality of the paper was confirmed, as well as the insulating film's resistance to chemical warfare agents, though indirectly.

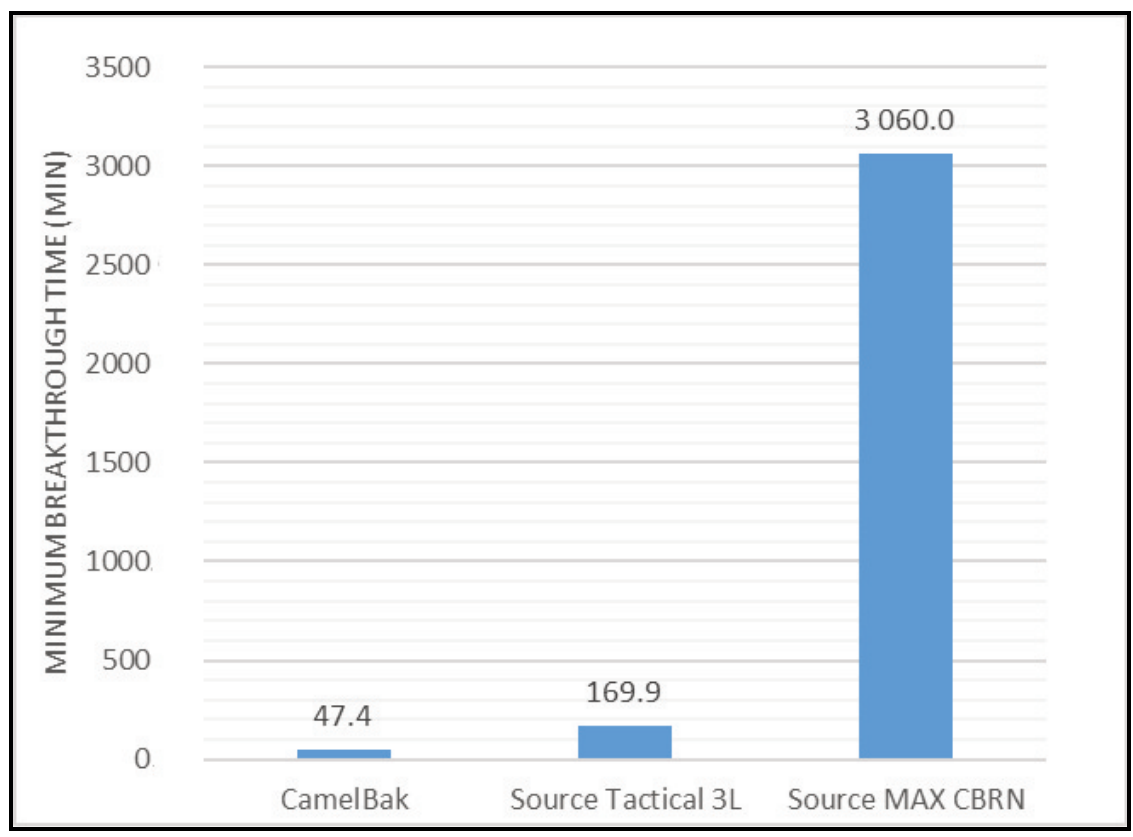

Fig. 3 Minimum breakthrough times of hydration bags films for liquid-phase mustard gas at a temperature of $30^{\circ} \mathrm{C}$

On the ground of assumptions that a direct contact with liquid-phase chemical warfare agents is rather unlikely and that an exposure to the vapours of these agents is more probable, the construction materials resistance was measured using the MINITEST method that enables to measure the material resistance against chemical warfare agents in the form of vapours. In view of theoretical assumptions that polymeric materials are 3-5 times more resistant to vapours of chemical warfare agents than to liquid-phase chemical warfare agents at the same temperature and material thickness, only the construction materials of the CamelBak and Source Tactical 3L hydration bags were tested. The minimum thickness values of the CamelBak and Source Tactical 3L hydration bags were $0.409 \mathrm{~mm}$ and $0.426 \mathrm{~mm}$, respectively. The measurement was performed at a temperature of $30^{\circ} \mathrm{C}$, and onto every measurement spot of the PVDF desk of the MINITEST device, $300 \mu \mathrm{l}$ of mustard gas was dosed. The minimum values of the breakthrough times measured were $174.6 \mathrm{~min}$ for the CamelBak hydration bag and $190.8 \mathrm{~min}$ for the Source Tactical 3L hydration bag. As 
for the CamelBak hydration bag, the breakthrough time for vapours was 3.68 times longer than for drops, by which the theoretical assumptions were confirmed. In case of the Source Tactical 3L hydration bag, there was not such increase. Repeated measurements with the particular material with a minimum thickness of $0.427 \mathrm{~mm}$ basically confirmed the results of the previous measurement, when a breakthrough time of 182.3 min was measured (Fig. 4).

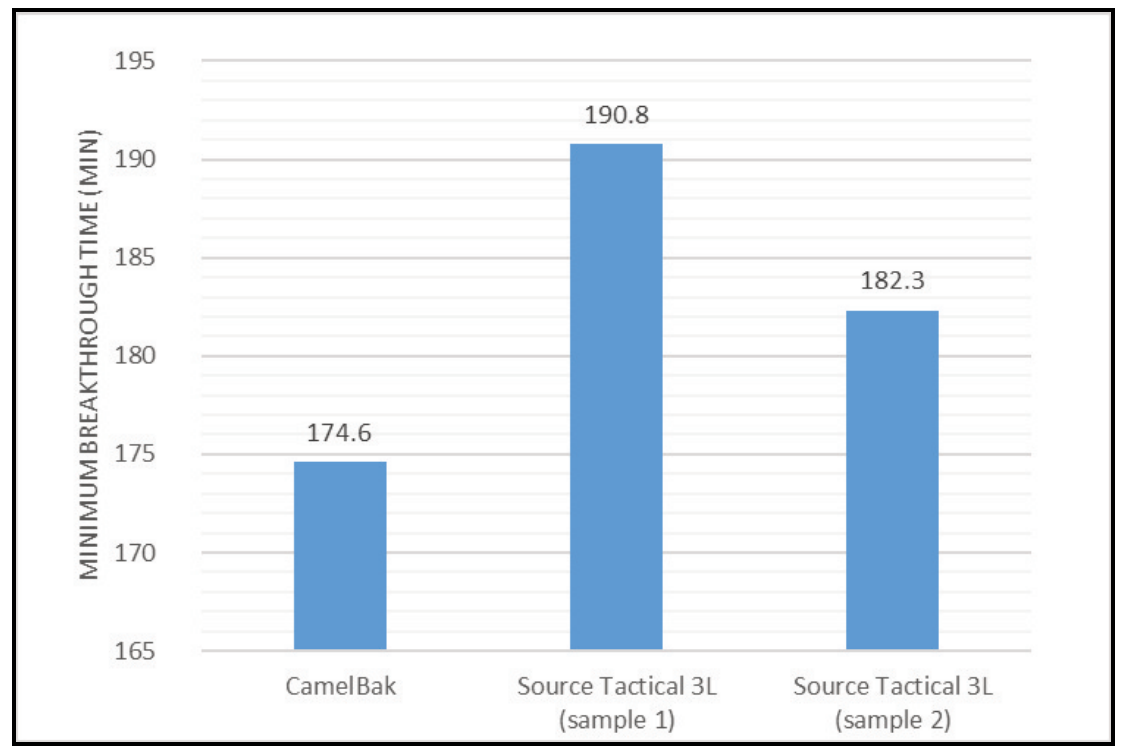

Fig. 4 Minimum breakthrough times of hydration bags for mustard gas vapours at a temperature of $30^{\circ} \mathrm{C}$

Due to the fact that the particular construction material type was unknown, the reason behind the results could be only speculated about. Although the bag was worn out as it had already been in use, this would not most probably lead to similar breakthrough times measured for the liquid-phase and vapour-phase mustard gas. When preparing the samples for measurement, it was discovered that the bag consisted of at least two layers which had been coming unstuck in several places. The breakthrough time measured there was $250 \mathrm{~min}$. Nevertheless, due to mechanical damage, i.e. the existence of interspace and the necessity to pass through it by means of diffusion mechanisms, these values were not included in the measurement results.

Since the vapours of chemical warfare agents must pass through the walls of backpacks that carry the hydration bags, the resistance of the individual layers as well as of their combination for mustard gas vapours was measured. It was assessed that it was not purposeful to measure their resistance for the drops due to infiltration by means of permeation or capillary effect with an immediate or almost immediate infiltration on the reverse side of the fabric layer. Only the backpack of the Army of the Czech Republic had its reverse side covered with a thin layer of polymeric material used to increase its impermeability to rainfall. The thickness of the fabrics was measured; however, they provided additional information only. As for the backpack of the Army of the Czech Republic (CamelBak), the minimum thickness of the outer fabric measured was $0.406 \mathrm{~mm}$, compared to that of the Source Tactical 3L which was $0.571 \mathrm{~mm}$. The minimum breakthrough times measured for mustard gas vapours at 
a temperature of $30^{\circ} \mathrm{C}$ using the MINITEST method were 9.6 min for the CamelBak fabric, $14.6 \mathrm{~min}$ for the Source Tactical $3 \mathrm{~L}$ outer fabric and $7.0 \mathrm{~min}$ for the Source MAX CBRN outer fabric (Fig. 5).

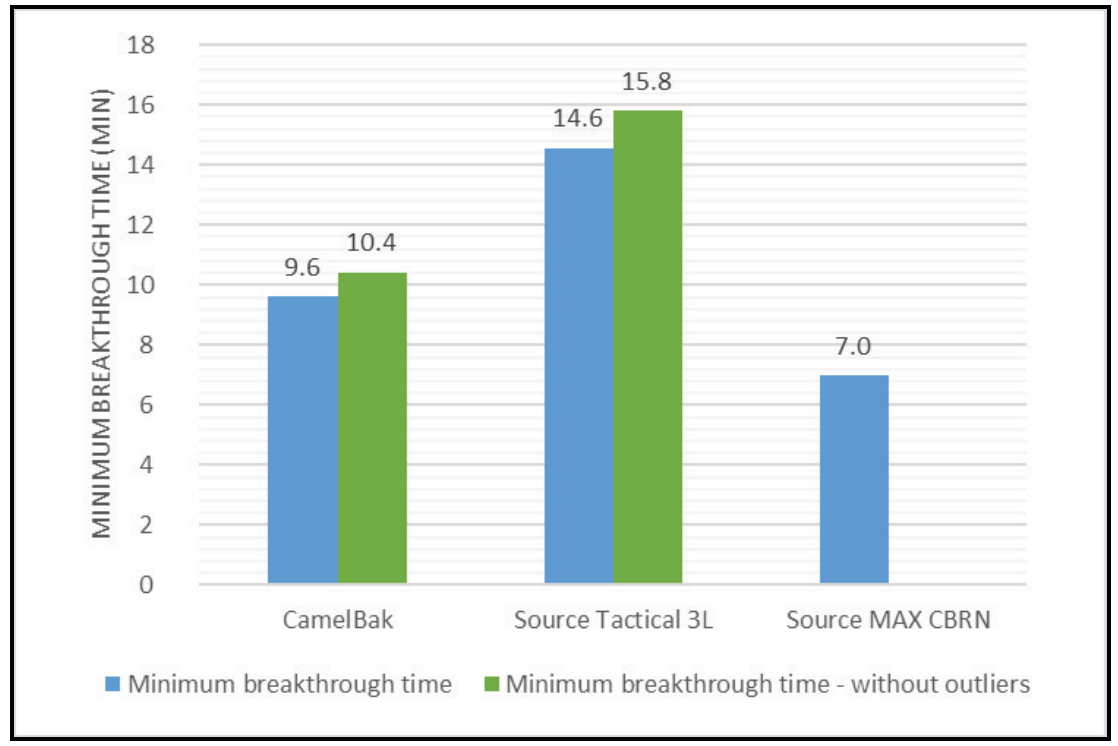

Fig. 5 Minimum breakthrough times of outer fabric of hydration bags backpacks for mustard gas vapours

Following the elimination of outliers, which could have been caused by structural nonuniformity of the material (Fig. 6), the breakthrough times of the outer fabric of the two first-mentioned backpacks did slightly increase. The fabric of the Source MAX CBRN 3L backpack was simultaneously permeated at all measurement spots. The polymeric layer on the reverse side of the backpack of the Army of the Czech Republic did not have any effect on the fabric's chemical resistance.

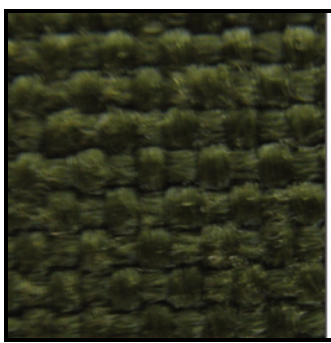

a)

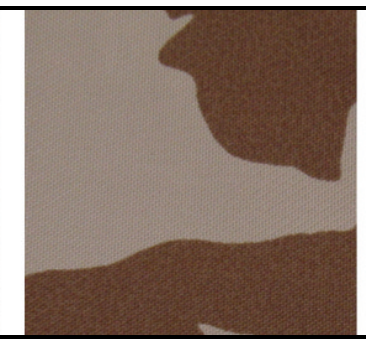

b)

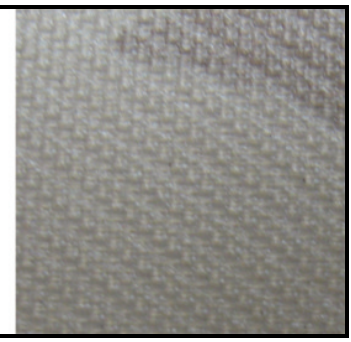

c)

Fig. 6 Outer fabric of hydration bags backpacks a) fabric of Source Tactical $3 L$,

b) outer side of fabric of backpack of the Army of the Czech Republic,

c) reverse side of fabric of backpack of the Army of the Czech Republic

The backpacks of the hydration bags consisted of inner and outer fabric inbetween which there was an insulating layer (Fig. 7). Using a micrometer, the thickness of all these layers of all backpacks was measured. Employing the MINITEST method, the breakthrough times for mustard gas vapours were measured. Considering 
the structure of individual layers, the measurement of breakthrough times for liquidphase mustard gas was not performed, as there was an assumption that mustard gas drops would quickly reach the reverse side of the backpack regardless of the layers' thickness. This assumption was confirmed by a quick permeation of textile layers by vapours, and there was an assumption that the inner insulating layer would react similarly.

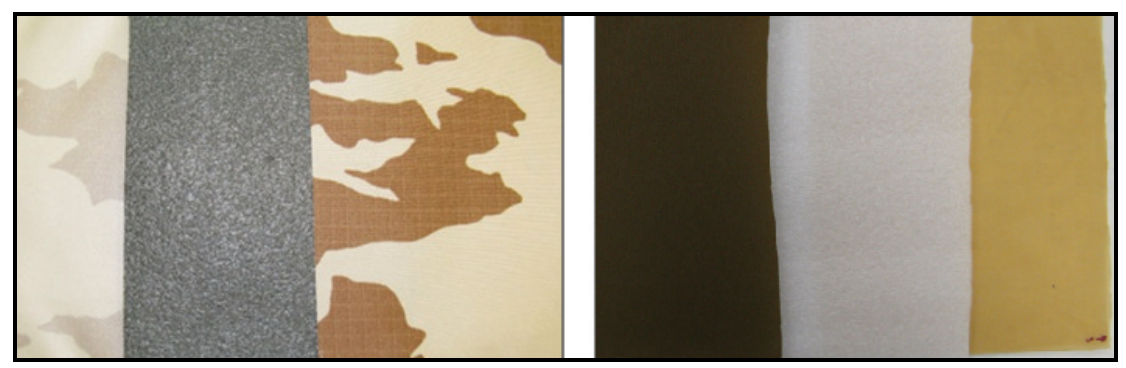

Fig. 7 Fabrics of backpack of the Army of the Czech Republic - left (dark layer - inner insulating foam layer) and of Source Tactical 3 L backpack - right (white layer - inner foam layer)

In Fig. 8, the minimum thickness values of the combination of layers of the hydration bags backpacks are shown. As shown there, the fabric of the backpack of the Czech Republic that carries the CamelBak hydration bag was the thickest, whereas the total minimum thickness values of the Source backpack were similar. The lower thickness of the Source Tactical 3L backpack could have been caused by its previous use, meaning that its thickness had changed as a result of compression, ageing etc.

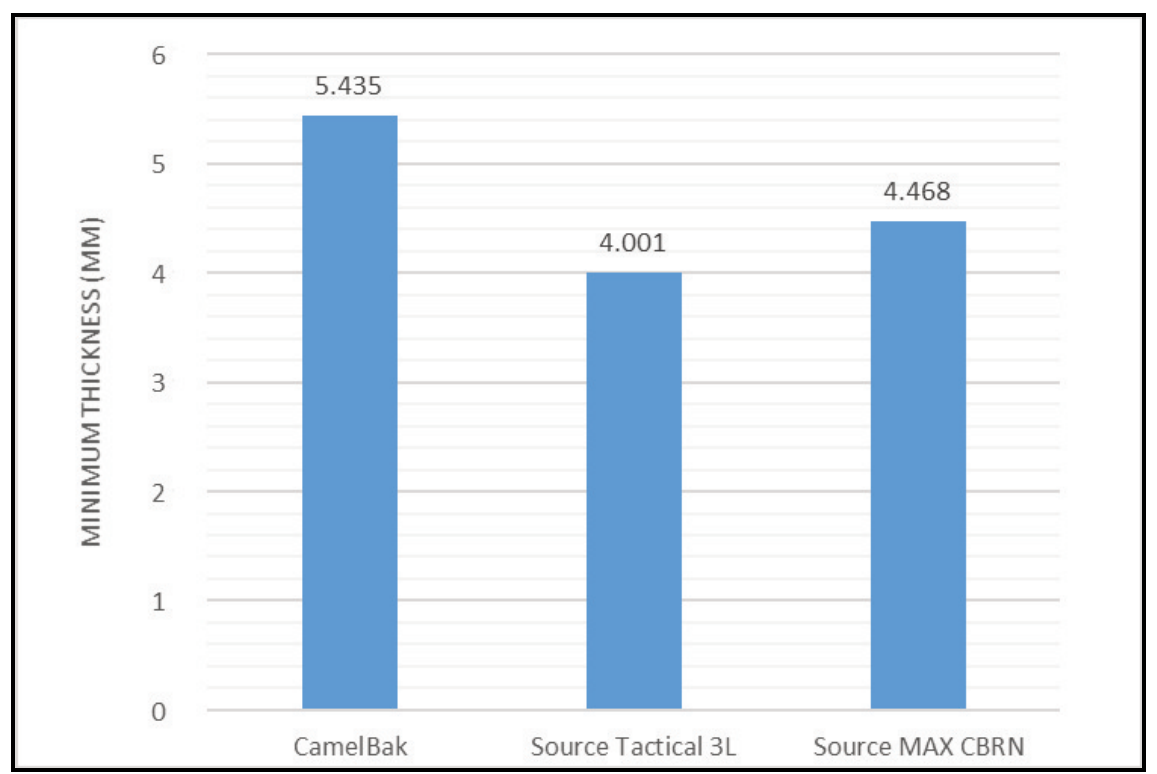

Fig. 8 Total minimum thickness of inner fabric, insulating layer and outer fabric combinations of hydration bags 
The measurement of breakthrough times has shown that that combination of textile layers and their thickness could have had a great impact on the permeation of chemical warfare agent also with equipment that is not designed for work in a contaminated area. As for the fabric combination of the backpack of the Army of the Czech Republic (CamelBak), the minimum breakthrough time measured was nearly $186 \mathrm{~min}$ (Fig. 9); contrastively, the minimum breakthrough times of the Source backpack were very alike $-30.9 \mathrm{~min}$ for the Source Tactical $3 \mathrm{~L}$ fabric combination and $40.3 \mathrm{~min}$ for the Source MAX CBRN fabric combination.

Since multi-layered materials with an interspace react differently than materials that are compact, the total breakthrough time of the combination of the backpack fabrics including the hydration bag film was tested. This was done by employing the MINITEST method used to measure their resistance to mustard gas vapours. The layers were compiled as they would be exposed to chemical warfare agents in a real contaminated area, which means that a combination of outer fabric, an insulating layer, inner fabric, and an insulating film of the hydration bag was tested. Only the water backpack of the Army of the Czech Republic with the CamelBak hydration bag (Fig. 10) and the Source Tactical 3L backpack were measured. The Source MAX CBRN 3L backpack was not measured, as it had already shown high resistance to liquid-phase mustard gas.

The minimum thickness values of the combination consisting of individual construction materials of the water backpack of the Army of the Czech Republic and the CamelBak hydration bag were $5.958 \mathrm{~mm}$ and $5.956 \mathrm{~mm}$ without outliers (Fig. 11), compared to the values of the Source Tactical 3L backpack which were $3.075 \mathrm{~mm}$ and $3.159 \mathrm{~mm}$ without outliers.

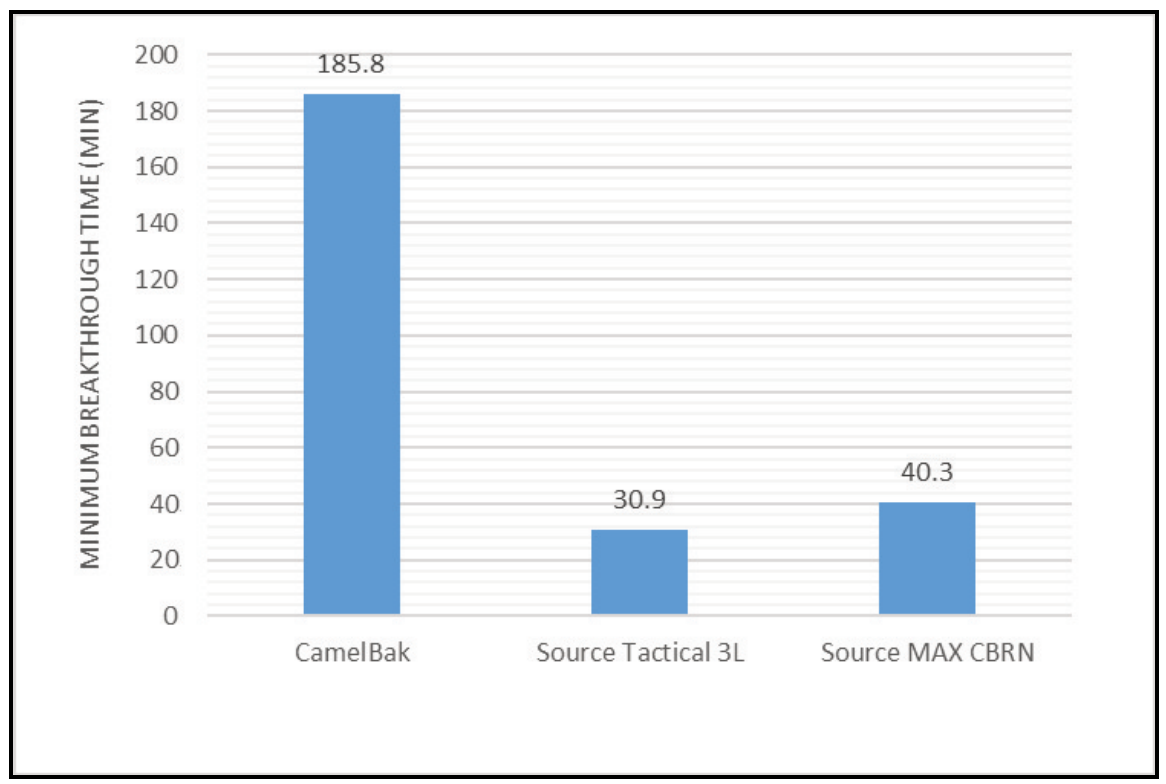

Fig. 9 Minimum breakthrough times of inner fabric, insulating layer and outer fabric combinations of hydration bags backpacks for mustard gas at a temperature of $30{ }^{\circ} \mathrm{C}$ using MINITEST method 


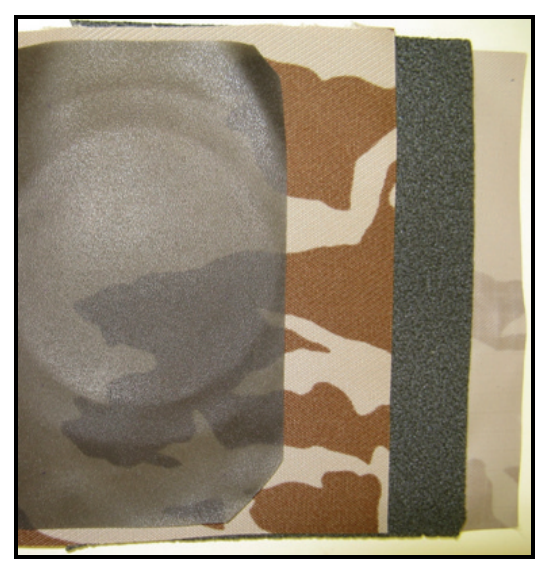

Fig. 10 Construction materials of water backpack of the Army of the Czech Republic and CamelBak hydration bag

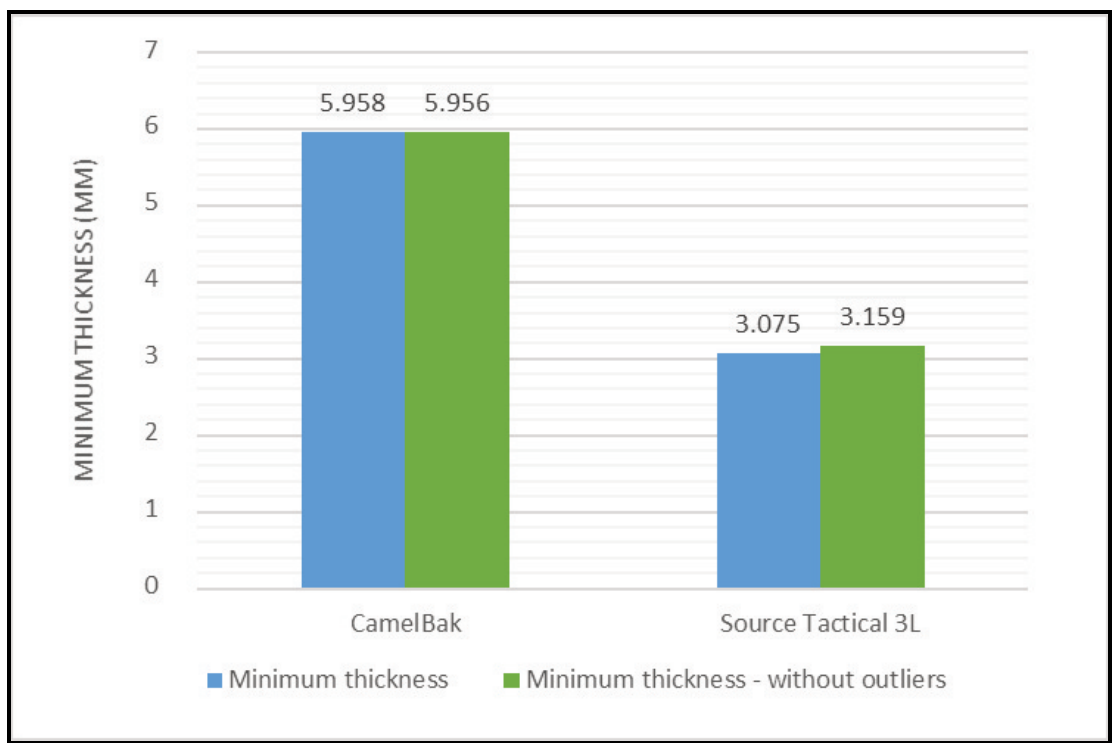

Fig. 11 Minimum thickness values of combination of construction materials for measurement of chemical resistance to mustard gas vapours

The minimum breakthrough times for mustard gas measured at a temperature of $30{ }^{\circ} \mathrm{C}$ were almost $540 \mathrm{~min}$ and nearly $553 \mathrm{~min}$ without outliers for the water backpack of the Army of the Czech Republic and the CamelBak hydration bag (Fig. 12). As for the Source Tactical 3L backpack, the minimum breakthrough times with and without outliers were similar - about $443 \mathrm{~min}$.

If the minimum breakthrough times of the textile parts of the backpacks and of the films of the hydration bags were basically added, the final breakthrough times would be 360.4 min for the water backpack of the Army of the Czech Republic with the CamelBak hydration bag and $221.7 \mathrm{~min}$ for the Source Tactical 3L hydration bag. When comparing these values with the minimum breakthrough time values of all 


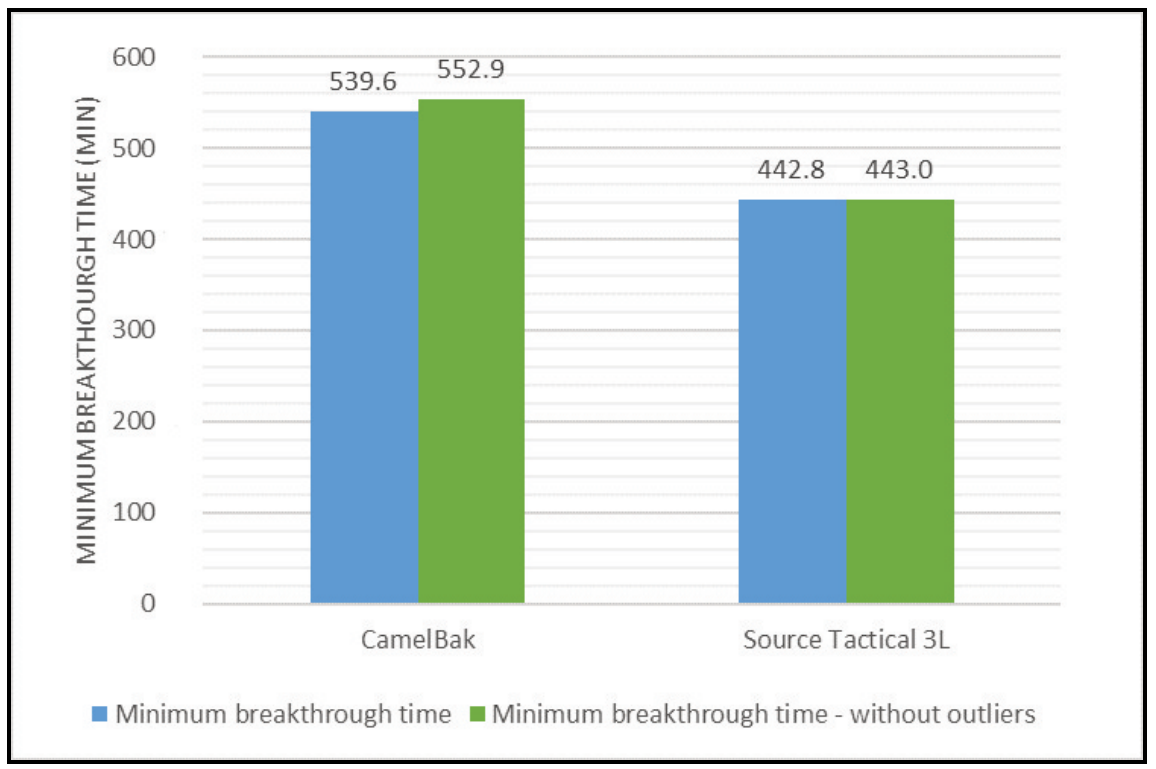

Fig. 12 Minimum breakthrough times of combinations for mustard gas vapours at a temperature of $30^{\circ} \mathrm{C}$ using MINITEST method

construction layers without eliminating outliers, the minimum breakthrough time of the water backpack of the Army of the Czech Republic with the CamelBak hydration bag would by higher by $49.7 \%$, whereas that of the Source Tactical 3L hydration bag would be higher by $99.7 \%$. It should be again noted that the Source Tactical $3 \mathrm{~L}$ had been in use for more than four years. Overall, construction materials as a whole offer long-time protection. This would be true provided that the backpacks were constructed in a way that the contaminated air could pass only through all of the construction layers. In fact, however, the backpacks include many spots where the air could pass freely and therefore have a direct effect on the hydration bag material.

Hydration bags include a suction tube through which water is brought directly from the bag into person's mouth or through a connecting adapter into the face mask. Since the suction tubes of the three hydration bags could be exposed to the direct effect of chemical warfare agents in a contaminated area, their chemical resistance was measured. The suction tubes of the CamelBak and the Source Tactical hydration bags had an outer textile layer that protects the tubes against mechanical damage but does not offer any chemical protection. The material of the suction tubes was fairly thick, and it was discovered that the tubes of the Source company consisted of two layers of material. Therefore, it was assumed that their breakthrough time would be long. Prior to the measurement, the textile layers were removed. It was observed, that the Source Tactical 3L hydration bag suction tube was probably covered in mould from the inside, and that the layers were coming unstuck in several places (Fig. 13).

Regardless of this fact, the suction tube's resistance to chemical warfare agents was measured. The measurement for liquid-phase mustard gas at a temperature of $30{ }^{\circ} \mathrm{C}$ confirmed the above given assumption about the suction tubes' high chemical resistance. As shown in Fig. 14, the measurement of the CamelBak and Source MAX CBRN 3L suction tubes` resistance was stopped after a period of $48 \mathrm{~h}$ (2880 min) 


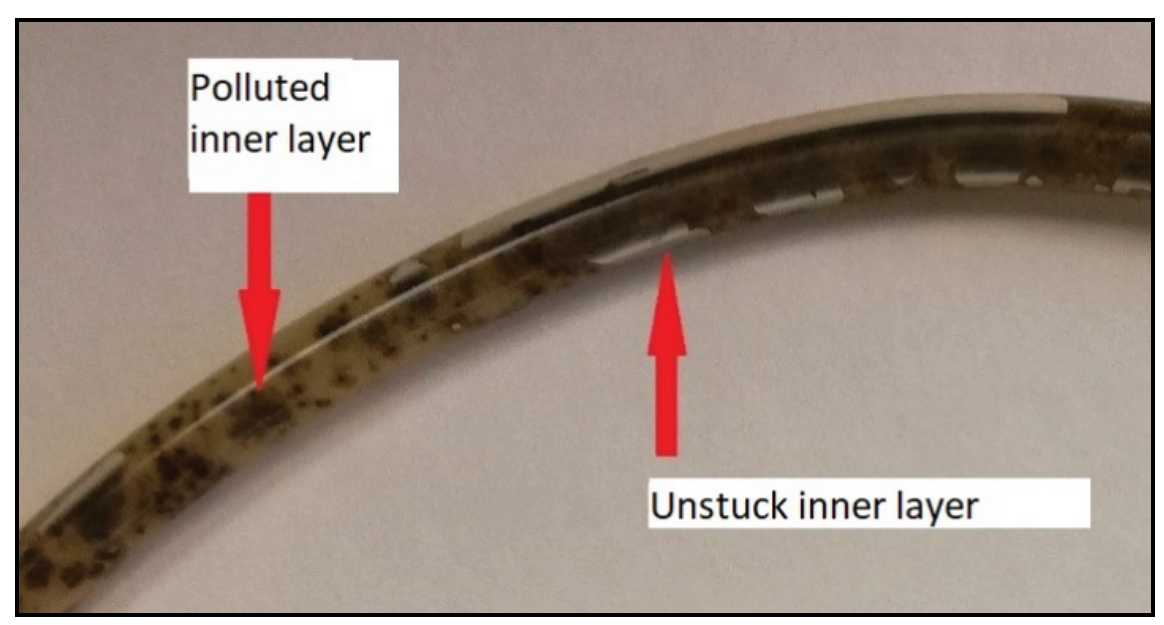

Fig. 13 Damage to the suction tube of the Source Tactical 3L hydration bag

without any permeation achieved. The minimum breakthrough time of the Source Tactical 3L was $464 \mathrm{~min}$. One of the Source Tactical 3L suction tube samples did not pass after $48 \mathrm{~h}$ and one did after a period of $1072 \mathrm{~min}$ After eliminating these outliers, the breakthrough time was $688.5 \mathrm{~min}$

It is hard to say how different the breakthrough time of this suction tube is from the one that is not damaged. Since its breakthrough time was more than $11 \mathrm{~h}$, the tube material showed good residual chemical resistance despite its previous usage and obvious damage.

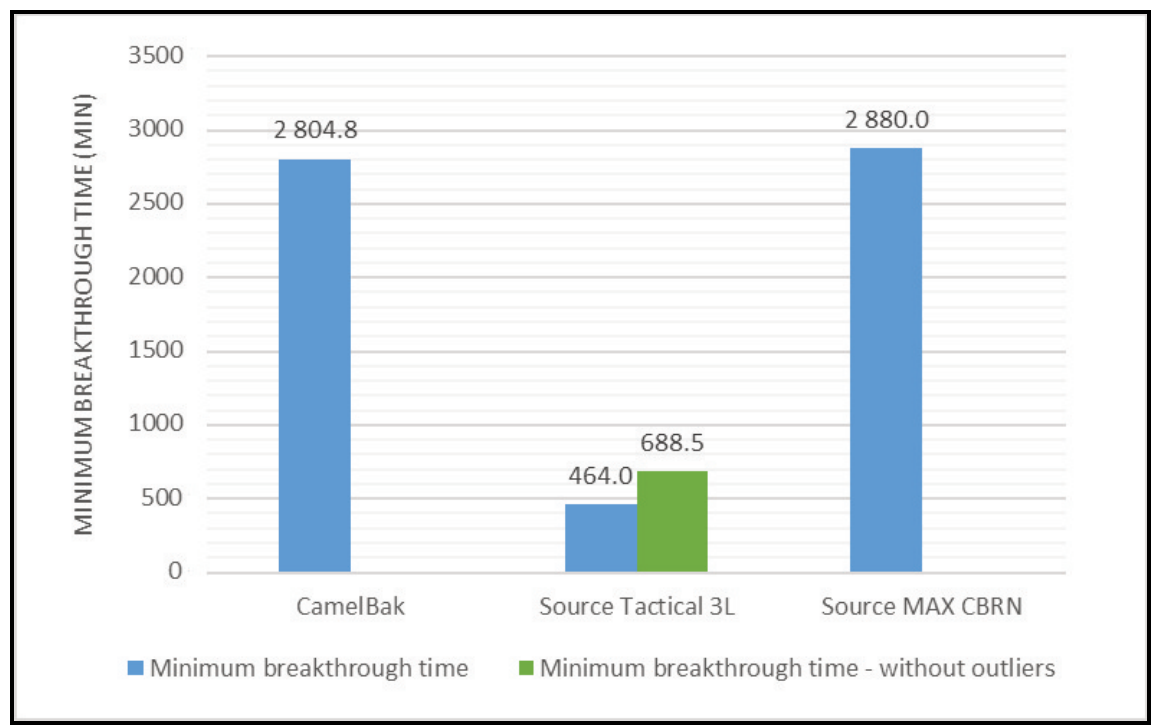

Fig. 14 Minimum breakthrough times of the suction tubes for liquid-phase mustard gas at a temperature of $30^{\circ} \mathrm{C}$ 


\section{Conclusion}

During a long-time work in a contaminated area using means of individual protection, hydration devices will become an integral part of equipment. It is essential to use such devices that would be safe and resistant to anticipated contaminant types, not only as far as their connection to the face mask is concerned, but also with regard to the resistance of their construction materials. Hydration device can be worn in various ways, on protective equipment or under it; however, it is necessary to modify equipment protecting the body surface, particularly regarding the latter given way of their wearing.

The experimental part has shown that means designed for work in an area contaminated by chemical warfare agents can be highly resistant and that their chemical resistance can greatly exceed the maximum period of time given by the manufacturers for a safe stay in the contaminated area. Nevertheless, means that are not primarily designed for work in a contaminated area can be resistant to the effects of chemical warfare agents for a long time as well. Backpacks consisting of several construction materials can significantly prolong the breakthrough time and thus enable its user to be hydrated when working in a contaminated area. These backpacks have a disadvantage, though - they do not form compact protective equipment and due to leakage issues, contaminated air can enter them easily. Understandably, if the hydration system is not designed for work in a contaminated area, it would most probably not be used in it. Constructional compactness should be a matter of course of hydration systems designed for work in contaminated areas.

There is another issue connected to the use of hydration bags and that is their decontamination. As for now, all of the examined hydration bags are meant to be used as disposable equipment. In case of the Source MAX CBRN hydration bag, it is stated by the manufacturer that the hydration bag is not designated to be decontaminated and it shall be disposed of in accordance with the procedures laid under national law. As for the other two hydration bags, the procedures should be conducted in the same way as the effect of the decontamination agents on the protective qualities of the bags is unknown.

\section{References}

[1] OTŘíSAL, P. and FLORUS, S. Application of Options of the QCM Detection Method for the Determination of Concentrations of Toxic Compounds Depending on Resistance Assessments of Barrier Materials (in Czech). Hygiena, 2013, vol. 58, no. 3, p. 125-129. ISSN 1802-6281.

[2] KLEINER, S.M. Water. Journal of the American Dietetic Association, 1999, vol. 99, no. 2, p. 200-206. DOI 10.1016/S0002-8223(99)00048-6.

[3] HUGO, J. and VOKURKA, M. Great Medical Dictionary (in Czech). $5^{\text {th }}$ ed. Prague: Maxdorf, 2005. 1008 p. ISBN 80-7345-058-5.

[4] Mayo Foundation for Medical Education and Research. Dehydration. [online]. [cited 2018-12-22]. Available from: http://mayocl.in/2rEQmaF.

[5] FLORUS, S, OTŘÍSAL, P. and HANZLÍK, V. Individual Protective Equipment and Collective Protection and their Use (in Czech). Prague: Ministry of Defence of the Czech Republic, 2014. 224 p. 
[6] Water Backpack (80501) (in Czech) [online]. S. P. M. Liberec spol. s r. o. 2012. [cited 2018-02-21]. Available from: http://bit.ly/2JbXEwQ.

[7] Source Tactical Gear. Tactical 3L Hydration pack [Datasheet]. Tirat Carmel: Source $^{\mathrm{TM}}$. Available from: http://bit.ly/2GFmDWV.

[8] Source Tactical Gear. MAX CBRN 3L Hydration pack [Datasheet]. Tirat Carmel: Source $^{\mathrm{TM}}$.

[9] FLORUS, S and OTŘÍSAL, P. Selected Methods of Study of Chemical Resistance of Insulation Protective Films for Chemical Warfare Agents (in Czech). Chemické listy, 2014, vol. 108, no. 9, p. 838-842. ISSN 0009-2770.

[10] OBŠEL, V, OTŘÍSAL, P. and FLORUS, S. The MINITEST Methodology for Determining Protective Properties of the Porous Materials Against Vapours of Sulphur Mustard and Selected Toxic Industrial Chemicals with the Possibility of Quick Statistical Evaluation of Obtained Results (in Czech) [Methodology]. Vyškov: CBRN Defence Institute, 2016. 20 p.

[11] FLORUS, S., OTŘÍSAL, P. and OBŠEL, V. Device for Testing of Resistance of Barrier Materials to Permeation of Vapours of Toxic Agents in Static Conditions (in Czech) [Utility Model]. Prague: Ministry of Defence of the Czech Republic, 2017, 30 479, CZ 30479 U1.

[12] OTRISAL, P., FLORUS, S., BARSAN G. and MOSTEANU, D. Employment of Simulants for Testing Constructive Materials Designed for Body Surface Isolative Protection in Relation to Chemical Warfare Agents. Revista de Chimie, 2018, vol. 69, no. 2, p. 300-304. ISSN 0034-7752.

[13] OTŘÍSAL, P. and FLORUS, S. Current and Perspectives in Personal and Collective Protection against Effects of Toxic Compounds (in Czech). Chemické listy, 2014, vol. 108, no. 12, p. 1168-1171. ISSN 0009-2770. 